\title{
A systematic review and meta-analysis of stereotactic body radiation therapy for colorectal pulmonary metastases
}

\author{
Christopher Cao ${ }^{1,2,3}$, Daniel Wang ${ }^{4}$, David H. Tian ${ }^{5,6}$, Ashley Wilson-Smith ${ }^{5}$, James Huang ${ }^{1}$, \\ Andreas Rimner ${ }^{7}$
}

${ }^{1}$ Department of Thoracic Surgery, Memorial Sloan Kettering Cancer Center, New York, USA; ${ }^{2}$ Department of Cardiothoracic Surgery, Royal Prince Alfred Hospital, Sydney, Australia; ${ }^{3}$ Chris O’Brien Lifehouse Hospital, Sydney, Australia; ${ }^{4}$ Department of Medicine, Cornell University, New York, USA; ${ }^{5}$ Collaborative Research Group, Macquarie University, Sydney, Australia; ${ }^{6}$ Department of Anaesthesia, Westmead Hospital, Sydney, Australia; ${ }^{7}$ Department of Radiation Oncology, Memorial Sloan Kettering Cancer Center, New York, USA

Contributions: (I) Conception and design: C Cao, A Rimner; (II) Administrative support: D Wang, DH Tian, A Wilson-Smith; (III) Provision of study materials or patients: None; (IV) Collection and assembly of data: D Wang, DH Tian, A Wilson-Smith; (V) Data analysis and interpretation: C Cao, DH Tian, J Huang, A Rimner; (VI) Manuscript writing: All authors; (VII) Final approval of manuscript: All authors.

Correspondence to: Christopher Cao. Department of Cardiothoracic Surgery, Royal Prince Alfred Hospital, Sydney, Australia; Chris O'Brien Lifehouse Hospital, Sydney, Australia. Email: drchriscao@gmail.com.

Background: There is growing evidence to support the hypothesis that radical treatment of pulmonary oligometastatic disease with stereotactic body radiation therapy (SBRT) can improve oncological outcomes. However, some reports suggest colorectal cancer (CRC) pulmonary metastases are associated with radioresistance. The present systematic review aimed to assess the local control (LC), overall survival (OS), and progression-free survival (PFS) of patients with CRC pulmonary metastases treated by SBRT. Secondary outcomes included assessment of peri-procedural complications and identification of prognostic factors on LC.

Methods: Electronic databases were systematically searched from their dates of inception using predefined criteria. Summative statistical analysis was performed for patients with CRC pulmonary metastases, and comparative meta-analysis was performed for patients with CRC versus non-CRC pulmonary metastases.

Results: Using predefined criteria, 18 relevant studies were identified from the existing literature. LC for CRC pulmonary metastases treated by SBRT at 1-, 2-, and 3-year were estimated to be $81 \%, 66 \%$, and $60 \%$, respectively. OS and PFS at 3 -year were $52 \%$ and $13 \%$, respectively. Patients with CRC pulmonary metastases were associated with significantly lower LC compared to non-CRC pulmonary metastases [HR, 2.93; 95\% confidence interval (CI), 1.93-4.45; $\mathrm{P}<0.00001$ ], but higher OS (HR, 0.61; 95\% CI, 0.45-0.82; $\mathrm{P}=0.001)$. There were no reported periprocedural mortalities and low incidences of periprocedural morbidities.

Conclusions: These findings may have implications for patient and treatment selection, dose fractionation, and support the hypothesis that CRC pulmonary metastases may require higher biological effective doses while respecting normal tissue constraints when treated with SBRT.

Keywords: Stereotactic body radiation therapy (SBRT); colorectal cancer (CRC); pulmonary metastasis; systematic review; meta-analysis

Submitted May 29, 2019. Accepted for publication Nov 15, 2019.

doi: $10.21037 /$ jtd.2019.12.12

View this article at: http://dx.doi.org/10.21037/jtd.2019.12.12

(c) Journal of Thoracic Disease. All rights reserved. 


\section{Introduction}

Stereotactic body radiation therapy (SBRT) has emerged as a safe and efficacious treatment modality for selected patients with pulmonary metastases $(1,2)$. Encouraging results from phase I and II trials have been followed by large multi-institutional databases to refine the patient selection process $(3,4)$. The increased utilisation of SBRT for patients with pulmonary metastases was reflected in a recent European survey involving 30 centres from six countries, in which $90 \%$ of the responding radiation oncology centres were treating pulmonary metastases with SBRT (5). There is now growing evidence to support the hypothesis that radical treatment of oligometastatic disease with SBRT can improve oncological outcomes (6-8).

Approximately $10-15 \%$ of all patients with colorectal cancer (CRC) develop pulmonary metastases, and CRC represents the second most common origin of all secondary pulmonary tumours $(2,4,9,10)$. Previous reports have demonstrated that the high doses per fraction delivered by SBRT have the ability to overcome differences in intrinsic radiosensitivity of different histologies in spinal metastases $(11,12)$. However, other reports suggest that SBRT for pulmonary metastases from colorectal origin may not achieve as high local control (LC) rates as pulmonary metastases of other primary histologies $(13,14)$. More recently, gene expression analyses have suggested an intrinsic radioresistance of colorectal pulmonary metastases, indicating that adjusted doses of SBRT may be necessary to achieve $\mathrm{LC}(15,16)$. These findings are somewhat surprising, as CRCs in their primary location are considered fairly radiosensitive and responsive to fractionated radiotherapy.

The primary objectives of the present systematic review were to assess the LC, overall survival (OS), and progression-free survival (PFS) outcomes of patients with CRC pulmonary metastases treated by SBRT. Metaanalysis was performed to compare these endpoints between patients with CRC pulmonary metastases and patients with non-CRC pulmonary metastases. Secondary outcomes included assessment of peri-procedural complications and identification of prognostic factors on local disease control.

\section{Methods}

\section{Literature search strategy}

The systematic review was performed using electronic databases EMBASE and Ovid Medline, from their dates of inception to November 2018. To ensure adequate sensitivity of the search, we combined the terms (pulm* or lung) and (metasta* or oligometasta*) and (sbrt or sabr or stereotactic body radiotherapy or stereotactic radiotherapy or radiosurgery) as either Medical Subject Headings or keywords. All identified articles were then assessed by applying the predefined selection criteria.

\section{Selection criteria and data appraisal}

Eligible studies for inclusion in the systematic review were those in which LC, PFS, or OS outcomes were presented for patients with colorectal pulmonary metastatic disease treated by SBRT. When institutions published duplicated studies with accumulating numbers of patients or increased lengths of follow-up, only the most complete or updated reports were included for statistical analysis. Case reports, conference abstracts or presentations, editorials, and publications not written in English were excluded. Studies with less than 10 patients were also excluded. Data were extracted from texts, tables, figures, and supplementary material. The definitions of LC, PFS, and OS were noted for each paper. Prognostic factors were selected based on categorization of the various prognosticators in each report. To assess the methodological quality of the selected studies, the Downs and Black scale was used to evaluate the quality index and categorized each report as good, fair or poor (17). Two investigators (D Wang and C Cao) independently reviewed each retrieved article. Discrepancies between the two reviewers were resolved by discussion and consensus.

\section{Statistical analysis}

Summative analysis was performed to examine two patient cohorts: (I) patients with colorectal pulmonary metastases identified from selected studies were analysed for LC, PFS, and OS; (II) when studies presented comparative outcomes for LC, PFS or OS for patients with colorectal pulmonary metastatic disease versus non-colorectal pulmonary metastases, these data were extracted and meta-analysed.

Meta-analysis was performed by combining the reported outcomes of selected studies using a random effect model. Hazard ratio (HR) and standard error were extracted or calculated from each study using the Tierney and Parmar methods described previously $(18,19)$. When calculations were not possible because of inadequate data, HRs were estimated using Kaplan-Meier graphs. $\mathrm{I}^{2}$ statistic was used to estimate the percentage of total variation across studies 


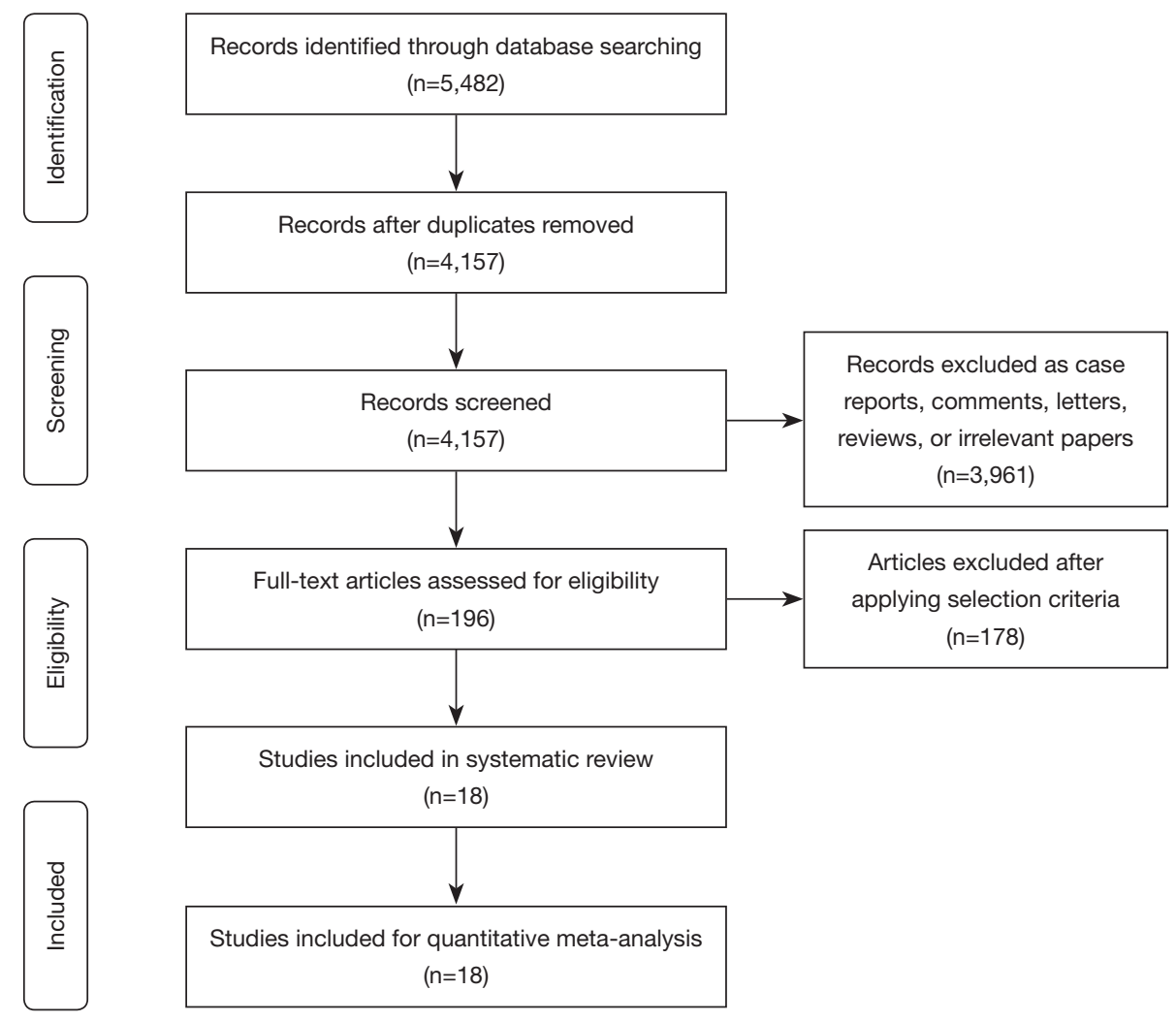

Figure 1 PRISMA flow chart summarizing the literature search strategy in the systematic review on stereotactic body radiation therapy for patients with colorectal and non-colorectal pulmonary metastases.

attributable to heterogeneity rather than chance. Metaanalysis was performed using Review Manager (version 5.1.2, Cochrane Collaboration, Oxford, United Kingdom). All $\mathrm{P}$ values were two-sided, and $\mathrm{P} \leq 0.05$ was considered to indicate statistical significance.

Individual patient survival data were reconstructed using Guyot's iterative algorithm to solve the KaplanMeier equations originally used to produce the published graphs (20). This algorithm used digitalized Kaplan-Meier curve data to find numerical solutions to the Kaplan-Meier equations, assuming a constant censoring mechanism. The reconstructed patient data were then aggregated to form the combined Kaplan-Meier curve. Reconstructed analyses were conducted using $\mathrm{R}$ (version 3.2.5, R Core Team, Vienna, Austria).

\section{Results}

\section{Quantity and quality of trials}

Applying the predefined selection criteria, a total of 5,482 records were found through the electronic search. After identification of additional records through other sources and removal of duplicate studies, 4,157 articles remained for screening. Of these, 3,961 were excluded on the basis of title or abstract content. After review of the full text of the remaining 196 articles, 18 were found to meet the selection criteria for the systematic review (13,21-37). All of the selected studies were non-randomized observational studies, with 9 studies presenting comparative clinical data on patients with colorectal pulmonary metastatic disease with non-colorectal pulmonary metastatic disease $(13,21,25,27-29,31,34,37)$. Quality assessment using the Downs and Black scale reported scores that indicated good $(13,21,23,24,26-28,35)$ or fair quality $(22,25,29-34,36,37)$. A summary of the study selection process is presented in the PRISMA chart in Figure 1. Patients were treated according to institutional regimens, with different doses, motion management, and beam management, as detailed in Table 1. Table S1 displays SBRT regimens, including Gy and fractions, by study. 
Table 1 Summary of baseline characteristics of patients who underwent stereotactic body radiation therapy for pulmonary metastases

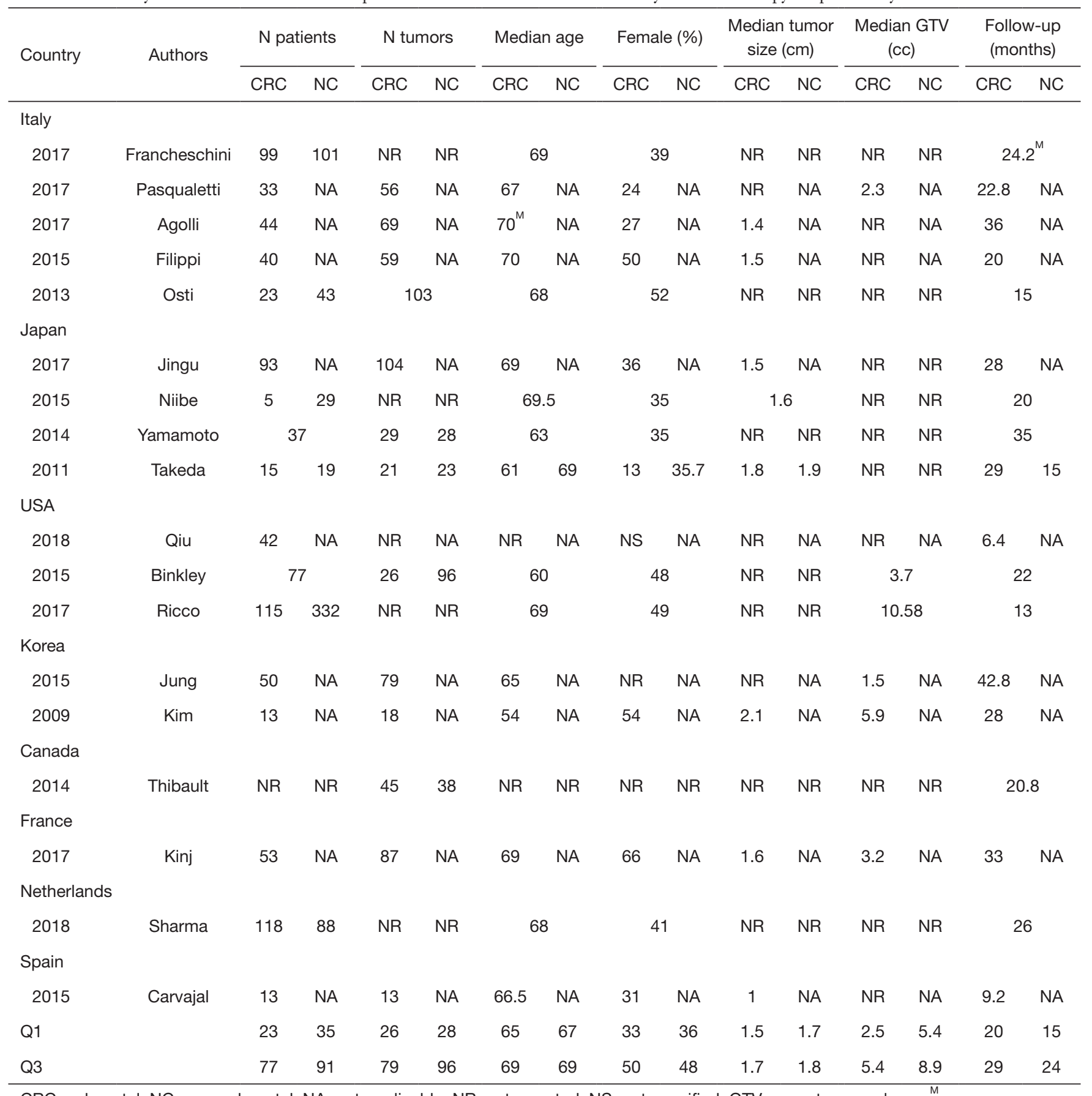

CRC, colorectal; NC, non-colorectal; NA, not applicable; NR, not reported; NS, not specified; GTV, gross tumor volume; ${ }^{\mathrm{M}}$, mean.

\section{Patient characteristics}

A summary of baseline patient characteristics is presented in Table 2. The median age was 68 years for CRC patients and 68.5 for non-CRC patients. The median percentage of females was $39 \%$ for CRC patients and $40 \%$ for non-CRC patients. Median length of follow-up was 23.5 months for CRC patients, and 20.8 months for non-CRC patients.

$L C$

LC was generally defined as the absence of growth within 
Table 2 Summary of baseline characteristics of patients who underwent stereotactic body radiation therapy for pulmonary metastases

\begin{tabular}{|c|c|c|c|c|c|c|c|c|c|c|c|c|c|c|c|}
\hline \multirow{2}{*}{ Country } & \multirow{2}{*}{ Authors } & \multicolumn{2}{|c|}{$\mathrm{N}$ patients } & \multicolumn{2}{|c|}{$\mathrm{N}$ tumors } & \multicolumn{2}{|c|}{ Median age } & \multicolumn{2}{|c|}{ Female (\%) } & \multicolumn{2}{|c|}{$\begin{array}{c}\text { Median tumor } \\
\text { size }(\mathrm{cm})\end{array}$} & \multicolumn{2}{|c|}{$\begin{array}{l}\text { Median GTV } \\
\text { (cc) }\end{array}$} & \multicolumn{2}{|c|}{$\begin{array}{c}\text { Follow-up } \\
\text { (months) }\end{array}$} \\
\hline & & CRC & $\mathrm{NC}$ & CRC & $\mathrm{NC}$ & CRC & $\mathrm{NC}$ & CRC & $\mathrm{NC}$ & CRC & $\mathrm{NC}$ & $\mathrm{CRC}$ & $\mathrm{NC}$ & $\mathrm{CRC}$ & $\mathrm{NC}$ \\
\hline \multicolumn{16}{|l|}{ Italy } \\
\hline 2017 & Francheschini & 99 & 101 & NR & NR & \multicolumn{2}{|c|}{69} & \multicolumn{2}{|c|}{39} & NR & NR & NR & NR & \multicolumn{2}{|c|}{$24.2^{M}$} \\
\hline 2017 & Pasqualetti & 33 & NA & 56 & NA & 67 & NA & 24 & NA & NR & NA & 2.3 & NA & 22.8 & NA \\
\hline 2017 & Agolli & 44 & NA & 69 & NA & $70^{M}$ & NA & 27 & NA & 1.4 & NA & NR & NA & 36 & NA \\
\hline 2015 & Filippi & 40 & NA & 59 & NA & 70 & NA & 50 & NA & 1.5 & NA & NR & NA & 20 & NA \\
\hline 2013 & Osti & 23 & 43 & \multicolumn{2}{|c|}{103} & \multicolumn{2}{|c|}{68} & \multicolumn{2}{|c|}{52} & NR & NR & NR & NR & \multicolumn{2}{|c|}{15} \\
\hline \multicolumn{16}{|l|}{ Japan } \\
\hline 2017 & Jingu & 93 & NA & 104 & NA & 69 & NA & 36 & NA & 1.5 & NA & NR & NR & 28 & NA \\
\hline 2015 & Niibe & 5 & 29 & NR & NR & \multicolumn{2}{|c|}{69.5} & \multicolumn{2}{|c|}{35} & \multicolumn{2}{|c|}{1.6} & NR & NR & \multicolumn{2}{|c|}{20} \\
\hline 2014 & Yamamoto & \multicolumn{2}{|c|}{37} & 29 & 28 & \multicolumn{2}{|c|}{63} & \multicolumn{2}{|c|}{35} & NR & NR & $\mathrm{NR}$ & NR & \multicolumn{2}{|c|}{35} \\
\hline 2011 & Takeda & 15 & 19 & 21 & 23 & 61 & 69 & 13 & 35.7 & 1.8 & 1.9 & NR & NR & 29 & 15 \\
\hline USA & & & & & & & & & & & & & & & \\
\hline 2018 & Qiu & 42 & NA & NR & NA & NR & NA & NS & NA & NR & NA & NR & NA & 6.4 & NA \\
\hline 2015 & Binkley & & & 26 & 96 & & & & & NR & NR & & & & \\
\hline 2017 & Ricco & 115 & 332 & NR & NR & & & & & NR & NR & & & & \\
\hline Korea & & & & & & & & & & & & & & & \\
\hline 2015 & Jung & 50 & NA & 79 & NA & 65 & NA & NR & NA & NR & NA & 1.5 & NA & 42.8 & NA \\
\hline 2009 & Kim & 13 & NA & 18 & NA & 54 & NA & 54 & NA & 2.1 & NA & 5.9 & NA & 28 & NA \\
\hline Canada & & & & & & & & & & & & & & & \\
\hline 2014 & Thibault & NR & NR & 45 & 38 & NR & NR & NR & NR & NR & NR & NR & NR & & \\
\hline France & & & & & & & & & & & & & & & \\
\hline 2017 & Kinj & 53 & NA & 87 & NA & 69 & NA & 66 & NA & 1.6 & NA & 3.2 & NA & 33 & NA \\
\hline Netherla & & & & & & & & & & & & & & & \\
\hline 2018 & Sharma & 118 & 88 & NR & NR & & & & & NR & NR & NR & NR & & \\
\hline Spain & & & & & & & & & & & & & & & \\
\hline 2015 & Carvajal & 13 & NA & 13 & NA & 66.5 & NA & 31 & NA & 1 & NA & NR & NA & 9.2 & NA \\
\hline Q1 & & 23 & 35 & 26 & 28 & 65 & 67 & 33 & 36 & 1.5 & 1.7 & 2.5 & 5.4 & 20 & 15 \\
\hline Q3 & & 77 & 91 & 79 & 96 & 69 & 69 & 50 & 48 & 1.7 & 1.8 & 5.4 & 8.9 & 29 & 24 \\
\hline
\end{tabular}

CRC, colorectal; NC, non-colorectal; NA, not applicable; NR, not reported; NS, not specified; GTV, gross tumor volume; ${ }^{\text {, }}$ mean.

the irradiated site. Overall, 15 studies with 686 colorectal pulmonary metastases were identified and analysed in the present systematic review for LC. A cumulative KaplanMeier graph is presented in Figure 2A, demonstrating an estimated 1-, 2-, and 3-year LC rate of $81 \%, 66 \%$, and
$60 \%$, respectively. Five studies provided comparative LC data for colorectal pulmonary metastases versus non-colorectal pulmonary metastases, with a forest plot demonstrating statistically significantly lower LC for colorectal pulmonary metastases [HR, 2.93; 95\% confidence 


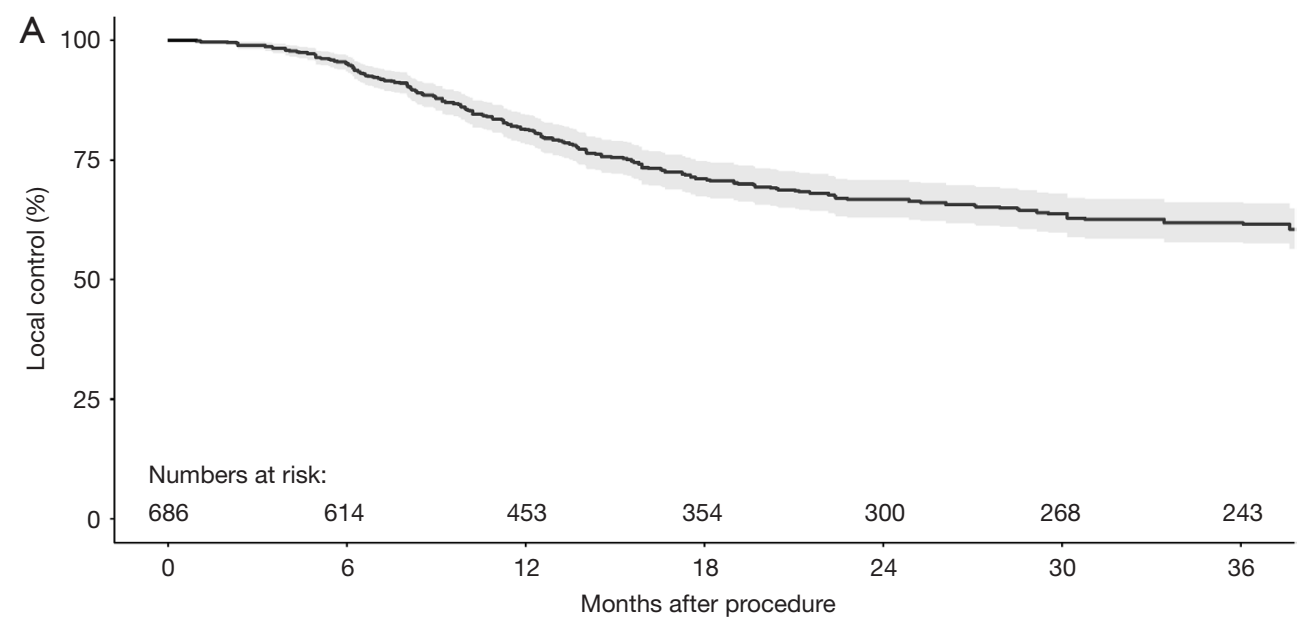

B

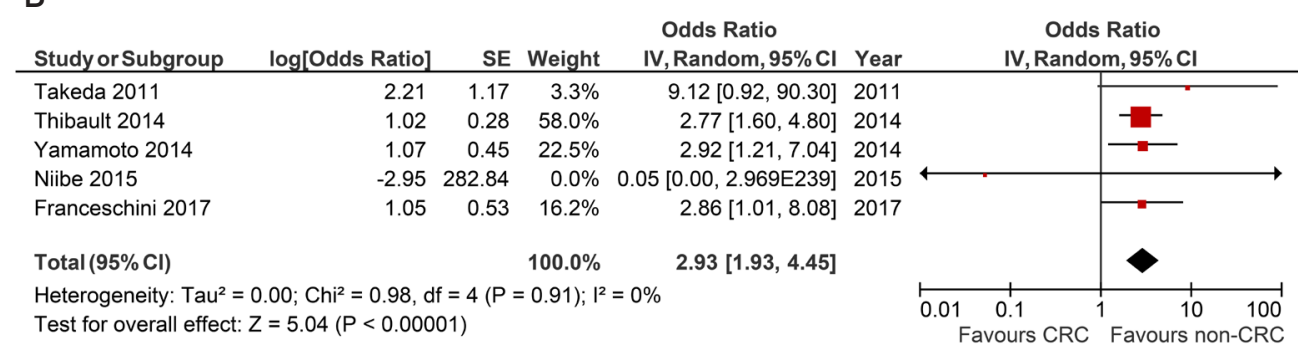

Figure 2 Local control. (A) Cumulative Kaplan-Meier graph demonstrating estimated local control of colorectal pulmonary metastases after stereotactic body radiation therapy. Shaded region indicates 95\% confidence interval (CI). (B) Forest plot of the odds ratio (OR) of local control in patients with colorectal pulmonary metastases versus non-colorectal pulmonary metastases after stereotactic body radiation therapy. The estimate of the OR of each study corresponds to the middle of the squares and the horizontal line shows the $95 \%$ CI. On each line, the numbers of events as a fraction of the total number randomized are shown for both treatment groups. For each subgroup, the sum of the statistics, along with the summary OR, is represented by the middle of the solid diamonds. A test of heterogeneity between the trials within a subgroup is given below the summary statistics.

interval (CI), 1.93-4.45; $\mathrm{P}<0.00001, \mathrm{I}^{2}=0 \%$, Figure 2B]. A summative reconstructed Kaplan-Meier graph comparing these two cohorts is shown in Figure 3 A.

\section{OS}

Eleven studies with 567 colorectal pulmonary metastases were analysed in the present systematic review for OS. The cumulative Kaplan-Meier graph is presented in Figure 3B, demonstrating an estimated 3-year OS rate of $52 \%$. All of the selected studies only included patients with oligometastatic disease, which was defined as five or fewer metastases in the specified studies. Three studies provided comparative OS data for colorectal pulmonary metastases versus non-colorectal pulmonary metastases, with a forest plot demonstrating statistically significantly increased
OS for colorectal pulmonary metastases (HR, 0.61; $95 \%$ CI, 0.45-0.82; $\mathrm{P}=0.001, \mathrm{I}^{2}=0 \%$, Figure $\left.3 B\right)$. A summative reconstructed Kaplan-Meier graph comparing these two cohorts is shown in Figure $4 A, B$.

\section{PFS}

PFS was generally defined as the lack of progression or relapse at any site after the commencement of SBRT. Six studies with 265 colorectal pulmonary metastases were analysed in the present systematic review for PFS. The cumulative Kaplan-Meier graph is presented in Figure 5, demonstrating an estimated 3 -year PFS rate of $13 \%$. There were an insufficient number of studies that compared colorectal pulmonary metastases versus non-colorectal pulmonary metastases for statistical analysis. 


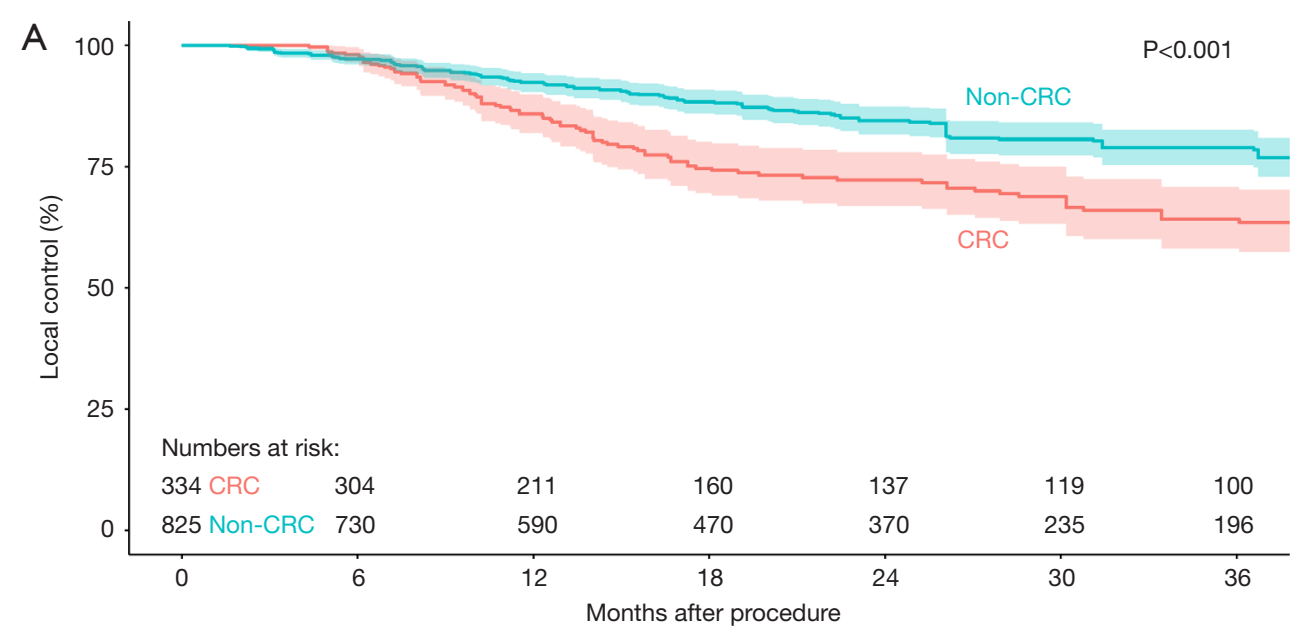

B

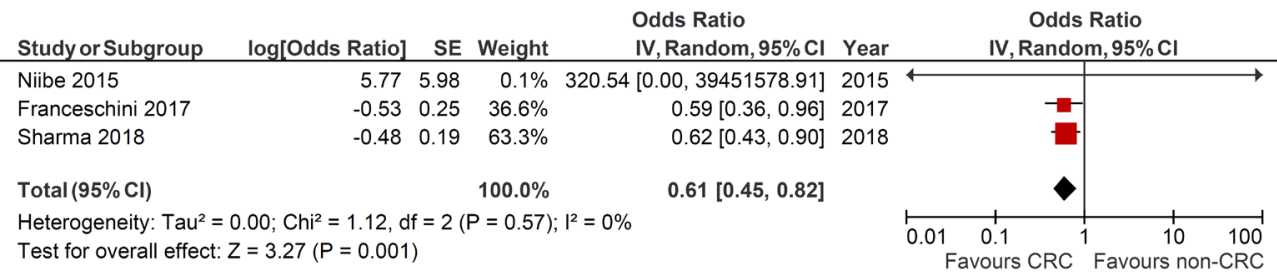

Figure 3 Local control. (A) Cumulative Kaplan-Meier graph demonstrating local control of colorectal pulmonary metastases versus noncolorectal pulmonary metastases after stereotactic body radiation therapy. Shaded regions represent $95 \%$ confidence interval (CI). (B) Forest plot of the odds ratio (OR) of local control in patients with colorectal pulmonary metastases versus non-colorectal pulmonary metastases after stereotactic body radiation therapy. The estimate of the OR of each study corresponds to the middle of the squares and the horizontal line shows the $95 \%$ CI. On each line, the numbers of events as a fraction of the total number randomized are shown for both treatment groups. For each subgroup, the sum of the statistics, along with the summary OR, is represented by the middle of the solid diamonds. A test of heterogeneity between the trials within a subgroup is given below the summary statistics.

\section{Mortality and morbidity}

There was no periprocedural mortality reported in any of the 18 studies identified in the present systematic review. Periprocedural morbidities included pneumonitis, esophagitis, dyspnoea, erythema, fatigue, and chest pain. The most common and serious complications included pneumonitis and dyspnoea, although their incidences were very low, as presented in Table 3.

\section{Prognostic factors}

A number of patient-, tumor- and treatment-related factors were identified from individual studies to have significant impact on local disease control. These included gender, age, histopathology, number and size of lesions, standardized uptake value (SUV) max, biologically effective dose (BED) of SBRT, and completeness of response. A summary of these prognostic factors is presented in Table $S 2$.

\section{Discussion}

With increased clinical experience, pulmonary metastases are increasingly being treated by SBRT with minimal peri-procedural toxicity. Previous studies have suggested differing oncological efficacies of SBRT based on the histology of the primary cancer $(13,28)$. More recently, multigene expression models have been developed to estimate the radiosensitivity index (RSI) of different tumor types $(15,38)$. Some have proposed the utilization of genomically-adjusted radiation dosing to personalize radiation therapy for patients with oligometastatic pulmonary metastases (39). Patients with radioresistant subtypes of lesions have been postulated to benefit from escalated BEDs, although these findings have largely been based on models using surgically resected specimens rather 

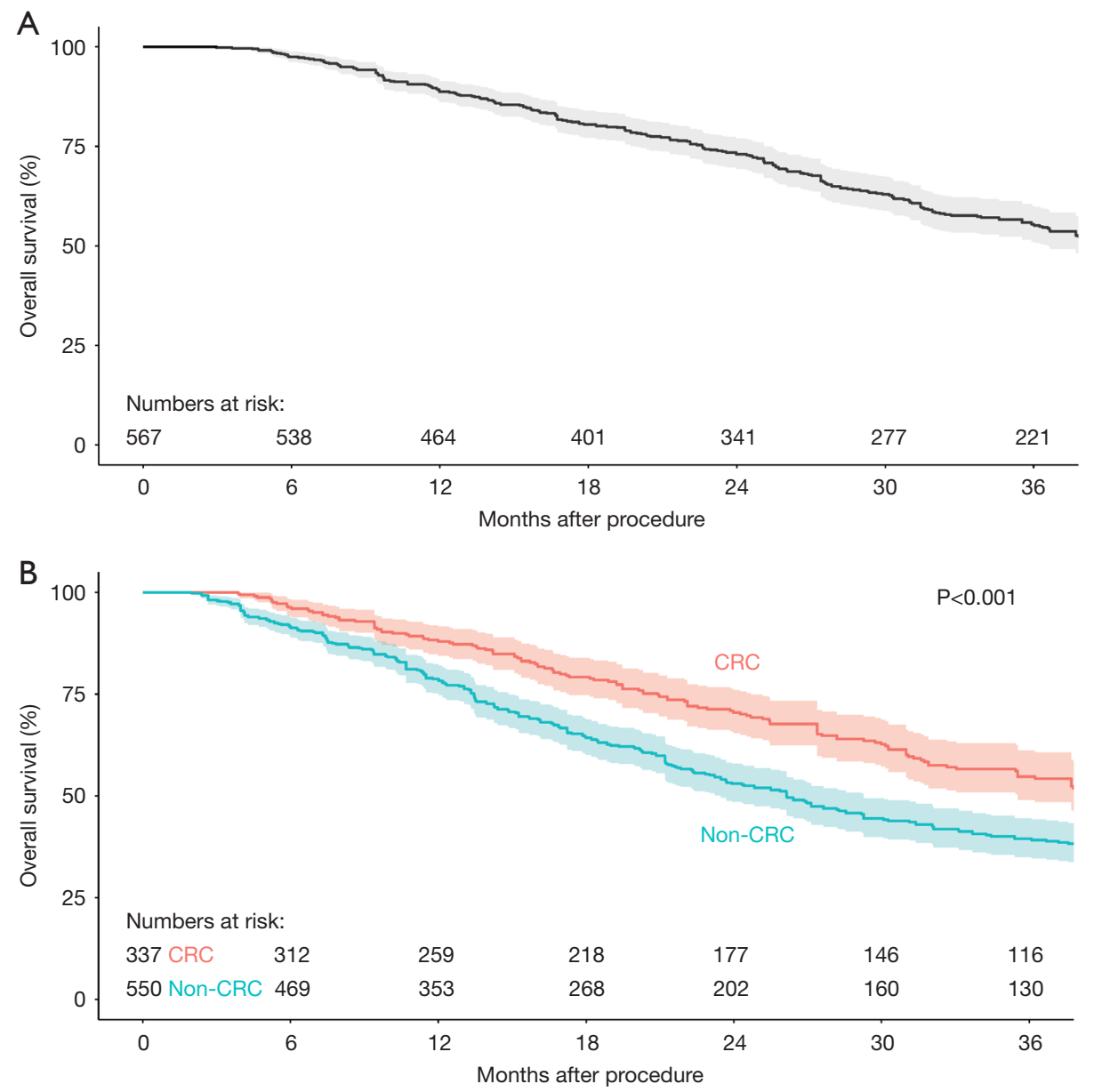

Figure 4 Overall survival. (A) Cumulative Kaplan-Meier graph demonstrating overall survival of patients with colorectal pulmonary metastases after stereotactic body radiation therapy. (B) Cumulative Kaplan-Meier graph demonstrating overall survival of patients with colorectal pulmonary metastases versus non-colorectal pulmonary metastases after stereotactic body radiation therapy. Shaded regions indicate $95 \%$ confidence interval.

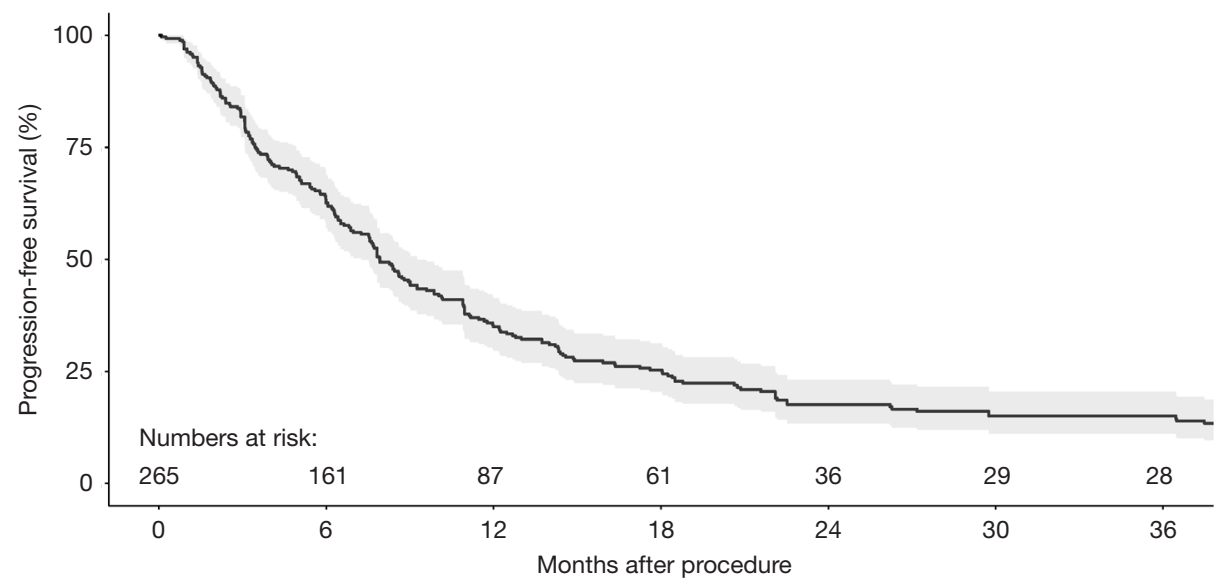

Figure 5 Progression-free survival. Shaded region indicates $95 \%$ confidence interval. 


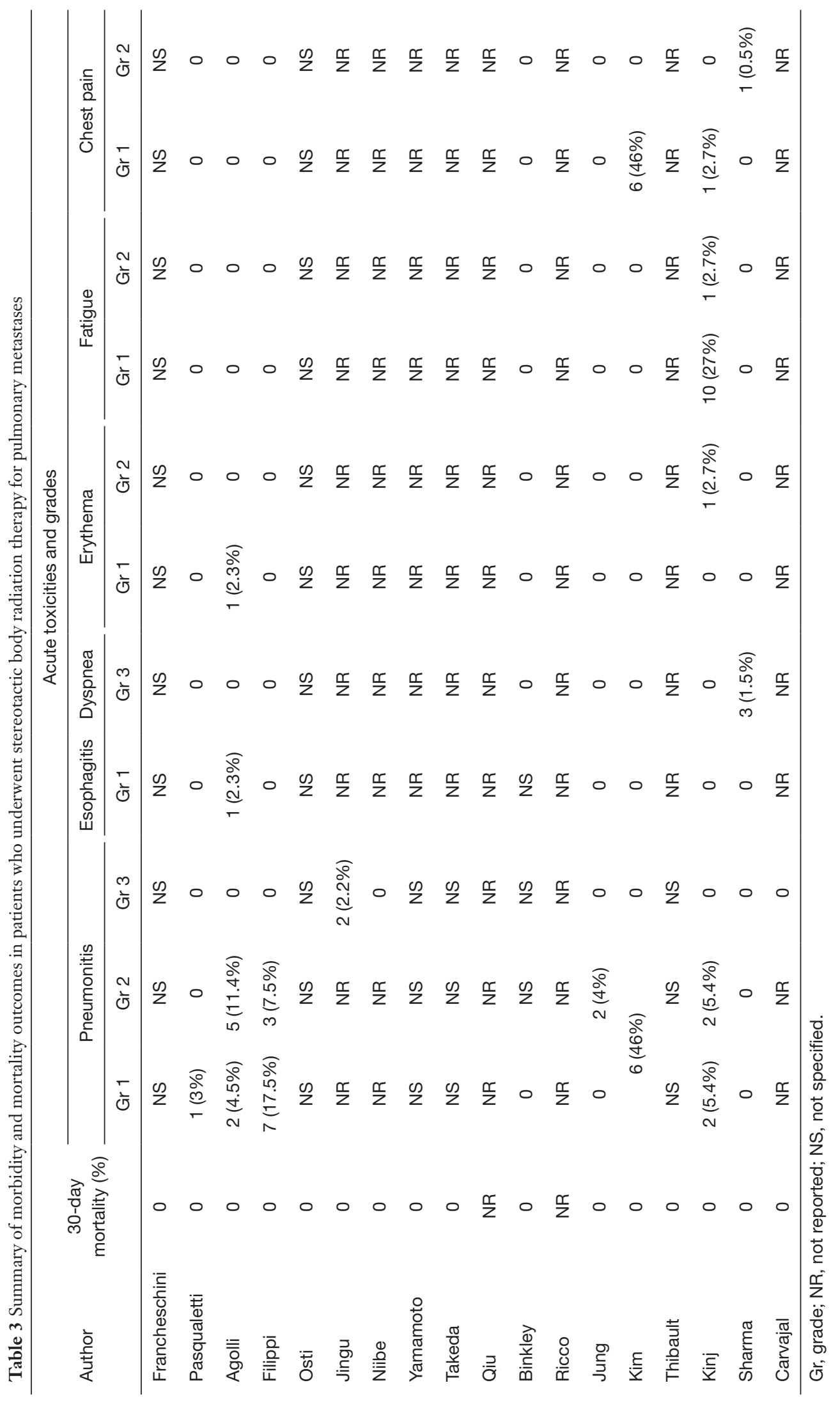


than direct RSI measurements (16).

The present systematic review identified 18 observational studies on patients with CRC pulmonary metastases that demonstrated 3-year LC, OS and PFS rates of $60 \%, 52 \%$, and $13 \%$, respectively. These findings may serve as useful benchmarks for future studies and help guide clinicians with their prognostic value. When using the available data to compare patients treated for CRC pulmonary metastases to non-CRC pulmonary metastases, there appeared to be significantly lower LC but higher OS for patients with CRC metastases. There was no reported periprocedural mortality, and the most common morbidities included pneumonitis, fatigue, and chest pain. A number of prognostic factors were found to be predictive of increased local recurrence for patients with CRC pulmonary metastases, such as increased size of metastatic lesions, increased number of lesions, and lower SBRT dosage. However, other prognostic factors were less consistent, such as colon versus rectal origin of the primary lesion, and the utilization of systemic therapy $(26,30,35)$.

Limitations of the present study included the variable treatment regimens prescribed in each institution, as well as differing baseline patient and tumour characteristics. The significance of adjuvant and neoadjuvant chemotherapy was difficult to interpret, partly due to the heterogenous regimens prescribed by each institution. Patient selection bias may also have caused some studies to report improved LC with adjuvant chemotherapy $(26,29)$, whilst others reported worse OS (30). Future prospective studies are required to improve the understanding of the role of systemic therapy in conjunction with SBRT for oligometastatic pulmonary metastatic disease. In addition, non-colorectal pulmonary metastases varied in histological origin and were sometimes not specified. Colonic and rectal cancers were often presented as a single group, and followup protocols also differed between studies. Nonetheless, the present systematic review represents the most comprehensive data to date, and provides a useful overview of oncological outcomes for patients with CRC metastases treated by SBRT.

In conclusion, SBRT in the treatment of colorectal pulmonary metastases has been shown to be safe, with no reported peri-procedural mortality and low rates of morbidity. Patients with colorectal pulmonary metastases are shown to have higher OS, but lower LC rates, when compared to patients with non-colorectal metastases. This supports previous findings that suggest an increased radioresistance of CRCs compared to pulmonary metastases of other primary histologies. These findings may have implications for patient and treatment selection, dose fractionation, or combination with systemic agents, and supports the hypothesis that patients with colorectal pulmonary metastases may require higher BED while respecting normal tissue constraints.

\section{Acknowledgments}

Funding: This research was funded in part through the NIH/NCI Cancer Center Support Grant P30 CA008748.

\section{Footnote}

Conflicts of Interest: The authors have no conflicts of interest to declare.

Ethical Statement: The authors are accountable for all aspects of the work in ensuring that questions related to the accuracy or integrity of any part of the work are appropriately investigated and resolved.

\section{References}

1. Navarria P, Ascolese AM, Tomatis S, et al. Stereotactic body radiotherapy (sbrt) in lung oligometastatic patients: role of local treatments. Radiat Oncol 2014;9:91.

2. Ricardi U, Filippi AR, Guarneri A, et al. Stereotactic body radiation therapy for lung metastases. Lung Cancer 2012;75:77-81.

3. Rusthoven KE, Kavanagh BD, Burri SH, et al. Multiinstitutional phase I/II trial of stereotactic body radiation therapy for lung metastases. J Clin Oncol 2009;27:1579-84.

4. Tanadini-Lang S, Rieber J, Filippi AR, et al. Nomogram based overall survival prediction in stereotactic body radiotherapy for oligo-metastatic lung disease. Radiother Oncol 2017;123:182-8.

5. Dahele M, Hatton M, Slotman B, et al. Stereotactic body radiotherapy: A survey of contemporary practice in six selected European countries. Acta Oncol 2015;54:1237-41.

6. Tree AC, Khoo VS, Eeles RA, et al. Stereotactic body radiotherapy for oligometastases. Lancet Oncol 2013;14:e28-37.

7. Gomez DR, Blumenschein GR Jr, Lee JJ, et al. Local consolidative therapy versus maintenance therapy or observation for patients with oligometastatic non-smallcell lung cancer without progression after first-line systemic therapy: a multicentre, randomised, controlled, 
phase 2 study. Lancet Oncol 2016;17:1672-82.

8. Iyengar P, Wardak Z, Gerber DE, et al. Consolidative Radiotherapy for Limited Metastatic Non-SmallCell Lung Cancer: A Phase 2 Randomized Clinical TrialConsolidative Radiotherapy for Limited Metastatic Non-Small-Cell Lung CancerConsolidative Radiotherapy for Limited Metastatic Non-Small-Cell Lung Cancer. JAMA Oncol 2018;4:e173501.

9. Mitry E, Guiu B, Cosconea S, et al. Epidemiology, management and prognosis of colorectal cancer with lung metastases: a 30-year population-based study. Gut 2010;59:1383-8.

10. Riihimäki M, Hemminki A, Sundquist J, et al. Patterns of metastasis in colon and rectal cancer. Sci Rep 2016;6:29765.

11. Yamada Y, Katsoulakis E, Laufer I, et al. The impact of histology and delivered dose on local control of spinal metastases treated with stereotactic radiosurgery. Neurosurg Focus 2017;42:E6.

12. Moussazadeh N, Lis E, Katsoulakis E, et al. FiveYear Outcomes of High-Dose Single-Fraction Spinal Stereotactic Radiosurgery. Int J Radiat Oncol Biol Phys 2015;93:361-7.

13. Binkley MS, Trakul N, Jacobs LR, et al. Colorectal Histology Is Associated With an Increased Risk of Local Failure in Lung Metastases Treated With Stereotactic Ablative Radiation Therapy. Int J Radiat Oncol Biol Phys 2015;92:1044-52.

14. Wang X, Zamdborg L, Ye H, et al. A matched-pair analysis of stereotactic body radiotherapy (SBRT) for oligometastatic lung tumors from colorectal cancer versus early stage non-small cell lung cancer. BMC Cancer 2018;18:962.

15. Ahmed KA, Fulp WJ, Berglund AE, et al. Differences Between Colon Cancer Primaries and Metastases Using a Molecular Assay for Tumor Radiation Sensitivity Suggest Implications for Potential Oligometastatic SBRT Patient Selection. Int J Radiat Oncol Biol Phys 2015;92:837-42.

16. Ahmed KA, Scott JG, Arrington JA, et al. Radiosensitivity of Lung Metastases by Primary Histology and Implications for Stereotactic Body Radiation Therapy Using the Genomically Adjusted Radiation Dose. J Thorac Oncol 2018;13:1121-7.

17. Downs SH, Black N. The feasibility of creating a checklist for the assessment of the methodological quality both of randomised and non-randomised studies of health care interventions. J Epidemiol Community Health $1998 ; 52: 377-84$.
18. Tierney JF, Stewart LA, Ghersi D, et al. Practical methods for incorporating summary time-to-event data into metaanalysis. Trials 2007;8:16.

19. Parmar MK, Torri V, Stewart L. Extracting summary statistics to perform meta-analyses of the published literature for survival endpoints. Stat Med 1998;17:2815-34.

20. Guyot P, Ades A, Ouwens MJ, et al. Enhanced secondary analysis of survival data: reconstructing the data from published Kaplan-Meier survival curves. BMC Med Res Methodol 2012;12:9.

21. Franceschini D, Cozzi L, De Rose F, et al. Role of stereotactic body radiation therapy for lung metastases from radio-resistant primary tumours. J Cancer Res Clin Oncol 2017;143:1293-9.

22. Pasqualetti F, Montrone S, Vivaldi C, et al. Stereotactic Body Radiotherapy in Patients with Lung Oligometastases from Colorectal Cancer. Anticancer Res 2017;37:315-9.

23. Agolli L, Bracci S, Nicosia L, et al. Lung Metastases Treated With Stereotactic Ablative Radiation Therapy in Oligometastatic Colorectal Cancer Patients: Outcomes and Prognostic Factors After Long-Term Follow-Up. Clin Colorectal Cancer 2017;16:58-64.

24. Filippi AR, Guerrera F, Badellino S, et al. Exploratory Analysis on Overall Survival after Either Surgery or Stereotactic Radiotherapy for Lung Oligometastases from Colorectal Cancer. Clin Oncol (R Coll Radiol) 2016;28:505-12.

25. Osti MF, Carnevale A, Valeriani M, et al. Clinical outcomes of single dose stereotactic radiotherapy for lung metastases. Clin Lung Cancer 2013;14:699-703.

26. Jingu K, Matsuo Y, Onishi H, et al. Dose Escalation Improves Outcome in Stereotactic Body Radiotherapy for Pulmonary Oligometastases from Colorectal Cancer. Anticancer Res 2017;37:2709-13.

27. Niibe Y, Yamashita H, Sekiguchi K, et al. Stereotactic Body Radiotherapy Results for Pulmonary Oligometastases: A Two-Institution Collaborative Investigation. Anticancer Res 2015;35:4903-8.

28. Yamamoto T, Jingu K, Shirata $Y$, et al. Outcomes after stereotactic body radiotherapy for lung tumors, with emphasis on comparison of primary lung cancer and metastatic lung tumors. BMC Cancer 2014;14:464.

29. Takeda A, Kunieda E, Ohashi T, et al. Stereotactic body radiotherapy (SBRT) for oligometastatic lung tumors from colorectal cancer and other primary cancers in comparison with primary lung cancer. Radiother Oncol 2011;101:255-9.

30. Qiu H, Katz AW, Chowdhry AK, et al. Stereotactic 
Body Radiotherapy for Lung Metastases from Colorectal Cancer: Prognostic Factors for Disease Control and Survival. Am J Clin Oncol 2018;41:53-8.

31. Ricco A, Davis J, Rate W, et al. Lung metastases treated with stereotactic body radiotherapy: the RSSearch(R) patient Registry's experience. Radiat Oncol 2017;12:35.

32. Jung J, Song SY, Kim JH, et al. Clinical efficacy of stereotactic ablative radiotherapy for lung metastases arising from colorectal cancer. Radiat Oncol 2015;10:238.

33. Kim MS, Yoo SY, Cho CK, et al. Stereotactic body radiation therapy using three fractions for isolated lung recurrence from colorectal cancer. Oncology 2009;76:212-9.

34. Sharma A, Duijm M, Oomen-de Hoop E, et al. Factors affecting local control of pulmonary oligometastases treated with stereotactic body radiotherapy. Acta Oncol 2018;57:1031-7.

35. Kinj R, Bondiau PY, Francois E, et al. Radiosensitivity of Colon and Rectal Lung Oligometastasis Treated With

Cite this article as: Cao C, Wang D, Tian DH, Wilson-Smith A, Huang J, Rimner A. A systematic review and meta-analysis of stereotactic body radiation therapy for colorectal pulmonary metastases. J Thorac Dis 2019;11(12):5187-5198. doi: 10.21037/ jtd.2019.12.12
Stereotactic Ablative Radiotherapy. Clin Colorectal Cancer 2017;16:e211-20.

36. Carvajal C, Navarro-Martin A, Cacicedo J, et al. Stereotactic body radiotherapy for colorectal lung oligometastases: preliminary single-institution results. J BUON 2015;20:158-65.

37. Thibault I, Poon I, Yeung L, et al. Predictive factors for local control in primary and metastatic lung tumours after four to five fraction stereotactic ablative body radiotherapy: a single institution's comprehensive experience. Clin Oncol (R Coll Radiol) 2014;26:713-9.

38. Eschrich SA, Pramana J, Zhang H, et al. A gene expression model of intrinsic tumor radiosensitivity: prediction of response and prognosis after chemoradiation. Int J Radiat Oncol Biol Phys 2009;75:489-96.

39. Scott JG, Berglund A, Schell MJ, et al. A genomebased model for adjusting radiotherapy dose (GARD): a retrospective, cohort-based study. Lancet Oncol 2017;18:202-11. 
Supplementary

Table S1 Summary of stereotactic body radiation therapy (SBRT) doses by study, for patients with colorectal and non-colorectal pulmonary metastases

\begin{tabular}{ll}
\hline Study & SBRT regimen (Gy/F) \\
\hline Sharma & $30 / 1,51-60 / 3-5,49 / 7,48-56 / 6-7$ \\
Franchescini & $30 / 1,32 / 4,36 / 6,48 / 4,54 / 3,60 / 3,60 / 8$ \\
Ricco & $48-54 / 1-8$ \\
Binkley & $25 / 1,50 / 4$ \\
Niibe & $48-50 / 4,35-80 / 7-10$ \\
Thibault & $50 / 5,52 / 4$ \\
Yamamoto & $40 / 4,45 / 3,46.5 / 4,48 / 4,50 / 8,56.2 / 8$, \\
Osti & $60 / 8,60 / 15$ \\
Takeda & $23 / 1,30 / 1$ \\
Qiu & $50 / 5$ \\
Jingu & $50 / 5,50 / 10$ \\
Kinj & $40-65 / 3-15$ \\
Pasqualetti & $25 / 1,60 / 3,50-75 / 5$ \\
Agolli & $24-26 / 1,27-42 / 3$ \\
Carvajal & $23 / 1,30 / 1,45 / 3$ \\
Filippi & $34 / 1,54 / 3,60 / 4,60 / 8$ \\
Jung & $26 / 1,45 / 3,48 / 4,55 / 5,60 / 8$ \\
Kim & $40 / 4,48 / 4,60 / 3,60 / 4$ \\
\hline Gy, Gray; & $39-51 / 3$ \\
\hline
\end{tabular}

Gy, Gray; F, fraction. 


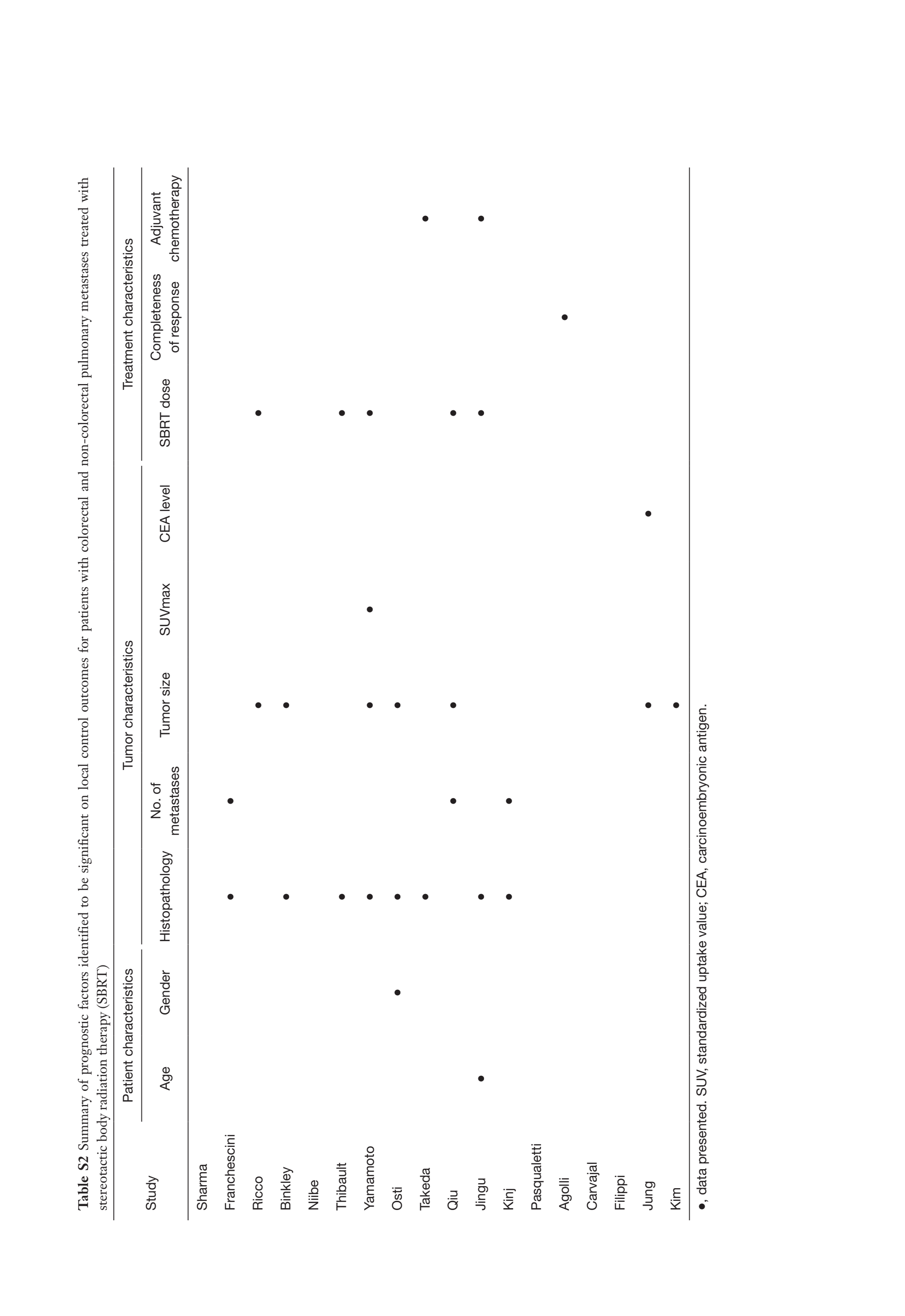

